\title{
Accreting millisecond X-ray pulsars: 10 years of INTEGRAL observations
}

\author{
M. Falanga $^{* 1}$, L. Kuiper ${ }^{2}$, J. Poutanen ${ }^{3}$, D. K. Galloway ${ }^{4}$, E. Bozzo ${ }^{5}$, A. Goldwurm ${ }^{6,7}$, W. \\ Hermsen $^{2}$, L. Stella ${ }^{8}$ \\ ${ }^{1}$ International Space Science Institute (ISSI), Hallerstrasse 6, CH-3012 Bern, Switzerland \\ ${ }^{2}$ SRON-Netherlands Institute for Space Research, Sorbonnelaan 2, 3584 CA, Utrecht, The \\ Netherlands \\ ${ }^{3}$ Astronomy Division, Department of Physics, P.O. Box 3000, FI-90014 University of Oulu, \\ Finland \\ ${ }^{4}$ Monash Center for Astrophysics, School of Physics, and School of Mathematical Sciences, \\ Monash University, VIC 3800, Australia \\ 5 ISDC - Universitè de Genève. Chemin d'Ecogia 16, CH-1290 Versoix, Switzerland \\ ${ }^{6}$ Service d'Astrophysique (SAp), IRFU/DSM/CEA-Saclay, 91191 Gif-sur-Yvette Cedex, France \\ ${ }^{7}$ Unité mixte de recherche Astroparticule et Cosmologie, 10 rue Alice Domon et Leonie Duquet, \\ F-75205 Paris, France \\ ${ }^{8}$ IINAF-Osservatorio Astronomico di Roma, via Frascati 33, 00040 Monteporzio Catone \\ (Roma), Italy \\ E-mail: mfalanga@issibern.ch
}

\begin{abstract}
During the last 10 years, INTEGRAL made a unique contribution to the study of accreting millisecond X-ray pulsars (AMXPs), discovering three of the 14 sources now known of this class. Besides increasing the number of known AMXPs, INTEGRAL also carried out observations of these objects above $20 \mathrm{keV}$, substantially advancing our understanding of their behaviour. We present here a review of all the AMXPs observed with INTEGRAL and discuss the physical interpretation of their behaviour in the X-ray domain. We focus in particular on the lightcurve profile during outburst, as well as the timing, spectral, and thermonuclear type-I X-ray bursts properties.
\end{abstract}

"An INTEGRAL view of the high-energy sky (the first 10 years)" 9th INTEGRAL Workshop and celebration of the 10th anniversary of the launch,

October 15-19, 2012

Bibliotheque Nationale de France, Paris, France

\footnotetext{
*Speaker.
} 


\section{Introduction}

The INTErnational Gamma-Ray Astrophysics Laboratory (INTEGRAL) was launched on 2002 October 17 to observe extreme astrophysical events in the gamma-ray sky. During the first ten years in orbit, INTEGRAL contributed to the discovery of three accreting millisecond X-ray pulsars (AMXPs): IGR J00291+5934 in 2004 [24, 6], IGR J17511-3057 in 2009 [8] , and IGR J174982921 in 2011 [9]. INTEGRAL also contributed to the understanding of two recently discovered AMXPs: HETE 1900.1-2455 in 2005 [7] and Swift J1749.4-2807 in 2010 [10]. XTE J1807294 was the first AMXP serendipitously observed with INTEGRAL IBIS/ISGRI between February and March 2003 [5]. The AMXP SAX J1808.4-3658 was also observed serendipitously from the beginning of its outburst in 2008 until back to quiescence (i.e., between September 18 and October 19, 2008). These INTEGRAL data still remain unpublished. The first outburst of the AMXP XTE J1751-305 was observed in April 2002 and lasted 12 days [17]. INTEGRAL observed the second outburst from this source in March 2005 [12], the third in April 2007 [2], and the fourth in October 2009 [1, 8]. The outbursts from XTE J1751-305 were all relatively short and similar to each other (see [23] and reference therein).

At present, 14 AMXPs are known. All these sources are X-ray transients, i.e. they spend most of the time in a quiescent phase (X-ray luminosities of $10^{31}-10^{33} \mathrm{erg} \mathrm{s}^{-1}$ ) and sometimes undergo X-ray outbursts reaching luminosities of $10^{36}-10^{37} \mathrm{erg} \mathrm{s}^{-1}$. During these events coherent pulsations are observed with frequencies in the range between 182 and $599 \mathrm{~Hz}$ (see e.g. [25, 19, 18] for a review). Since the launch in 2002, INTEGRAL provided a contribution to our understanding of the AMXPs by performing at least one observation of these objects per year. In this contribution we review all the INTEGRAL results on the AMXPs achieved after ten years in orbit.

\section{The INTEGRAL imaging of AMXPs}

Thanks to the unprecedented INTEGRAL capabilities to image the hard X-ray sky at relatively high angular resolutions ( $12^{\prime}$ for ISGRI and $3^{\prime}$ for JEM-X), all AMXPs could be clearly identified in the mosaicked images at discovery and their X-ray emission unambiguously disentangled from that of other nearby hard X-ray emitters [5, 6, 7, 8, 10, 9]. An example of mosaicked sky image around an AMXP in outburst is shown in Fig. 1 (left panel).

These capabilities were particularly relevant for the observation of the source IGR J00291+5934, which is located $\sim 18^{\prime}$ from the close-by persistent intermediate polar V609 Cas [6]. The AMXP source XTE J1751-305 underwent a short period of enhanced X-ray activity during the decay of the outburst from the AMXP IGR J17511-3057 located 20' away from the former object [8]. In the case of the AMXP IGR J17498-2921 INTEGRAL was able to disentangle the contributions of the surrounding objects and to clearly distinguish between the type-I X-ray bursts emitted by the source and those going off in the near-by bursters SLX 1744-300/299 and 1A 1742-294 [9]. These sources are located at $0.9^{\circ}$ and $0.86^{\circ}$ from the IGR J17498-2921 position (see Fig. 1; left panel).

\section{The AMXP outburst lightcurves}

Most of the AMXPs displayed in the past very similar behaviours while in outburst. A representative lightcurve of an outburst from an AMXP is shown in Fig. 1 (right panel). The lightcurves 

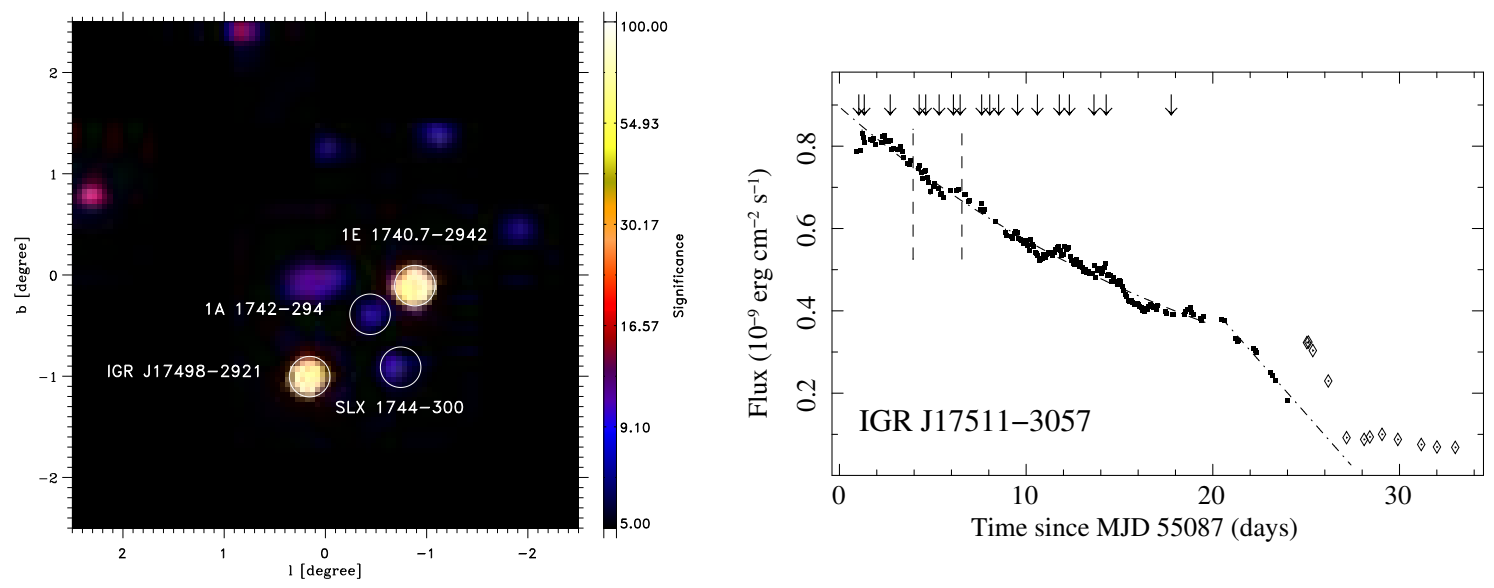

Figure 1: Left: INTEGRAL/ISGRI sky image of the field of view around IGR J17498-2921 in the 20-100 keV band for an effective exposure of $210 \mathrm{ks}$ (see [8]). Right: RXTE/PCA (2-20 keV) outburst light curve of IGR J175113057. The diamonds correspond to observations in which both XTE J1751-305 and IGR J17511-3057 were active. The arrows indicate the times of the detected X-ray bursts. The vertical dashed lines indicate the interval of the INTEGRAL observations (see [9]).

of these events are usually characterized by a fast rise (a few days at the most) and an exponential decay. The latter terminates with a break, after which a linear decay of the source X-ray flux is observed down to the quiescence level (see e.g., $[5,6,7,8,10,9]$ ). These outburst profiles are commonly interpreted in terms of the disk instability model, taking into account the irradiation of the disk by the central X-ray source [14]. This model has been applied to a sample of different LMXBs, including six AMXPs [22, 8, 10,9]. In these works it was shown that the timescale of the decay light curve and its luminosity at a characteristic time are linked to the outer radius of the accretion disk. The break ("knee") observed during the X-ray flux decay at the end of the outburst is believed to be a consequence of mass transfer onto the outer edge of the disk, as this supply of material to the compact object is effectively cut-off when the outer disk enters the cool low-viscosity state. The knee occurs at the lowest value of the X-ray luminosity at which the outer disk edge can still be kept hot by central illuminating source. The measured value of this luminosity can be used to estimate for the outer disk radius. A second independent estimate of this radius is given by the timescale of the exponential decay of the lightcurve.

\section{The AMXP X-ray spectra}

The available INTEGRAL JEM-X+ISGRI broad-band X-ray spectra of all AMXPs (XTE J1807-294, IGR J00291+5934, HETE 1900.1-2455, IGR J17511-3057, Swift J1749.4-2807, and IGR J17498-2921), can be usually well fit by using a thermal Comptonization model comprs [21] in the slab geometry $[5,6,7,8,10,9]$. The main model parameters are the Thomson optical depth $\tau_{\mathrm{T}} \sim 1-2$ across the slab, the electron temperature $k T_{\mathrm{e}} \sim 25-50 \mathrm{keV}$, the temperature $k T_{\mathrm{bb}} \sim 0.5-1.0 \mathrm{keV}$ of the soft-seed blackbody photons assumed to be injected from the bottom of the slab, the emission area $A_{\mathrm{bb}} \sim 20 \mathrm{~km}^{2}$. In Fig. 2 (left panel) we show a representative AMXP unfolded spectrum and the corresponding best-fit comprs model. 

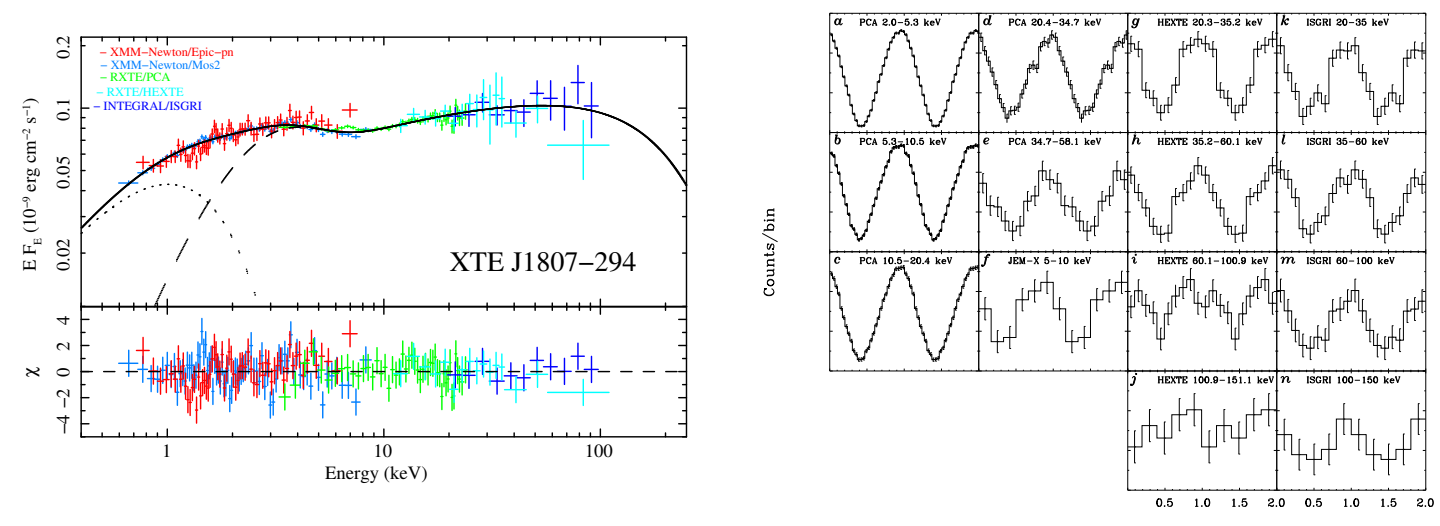

Pulse Phase

Figure 2: Left: Simultaneous INTEGRAL, XMM-Newton, and RXTE spectra of XTE J1807-294 fitted with an absorbed disk black body, DISKвB, plus comprs model (between March 20-22, 2003). The EPIC-pn and MOS2 spectra in the 0.5$10 \mathrm{keV}$ range and PCA/HEXTE in the $3-200 \mathrm{keV}$ and IBIS/ISGRI in the 20-200 keV range are shown. The DISKBв model is shown by a dotted curve, the dashed curve represents the comprs model, while the total spectrum is shown by a solid line. The lower panel presents the residuals from the best fit. See [6] for more details. Right: Pulse-profile collage of IGR J00291+5934 using data from RXTE/PCA (2-58 keV; panels a-e), RXTE/HEXTE (20-151 keV; panels g-j), INTEGRAL/JEM-X (5-10 keV; panel f) and INTEGRAL/ISGRI (20-150 keV; panels k-n). Two cycles are shown for clarity. All profiles have high off-set values due to the intrinsic properties of the instruments. The error bars represent 1 sigma statistical errors. All profiles reach their maximum near phase $\sim 0.95$. It is noteworthy the highly sinusoidal shape of the profiles for energies up to $100 \mathrm{keV}$.

The spectra of AMXPs are very similar to those of the so-called "atoll" sources at low luminosities, where the X-rays are probably produced in the boundary/spreading layer near the NS equator (see e.g., [21]). These similarities can be explained by assuming that in both classes of sources the energy dissipation occurs in an optically thin medium (i.e. the accretion shock and boundary/spreading layer) and the spectral properties are determined solely by energy balance and feedback from the NS surface (which in turns provides cooling in the form of soft photons).

\section{The AMXP timing above $20 \mathrm{keV}$}

In recent years, INTEGRAL proved to be crucial to perform timing analysis of the high-energy emission from AMXPs in the hard X-ray domain ( $>20 \mathrm{keV})$. In the case of IGR J00291+5934, IGR J17511-3057, and IGR J17498-2921, INTEGRAL/ISGRI was capable of measuring the pulse profile at frequencies of $1.67 \mathrm{~ms}, 2.5 \mathrm{~ms}$, and $4.08 \mathrm{~ms}$, respectively up to energies of $150 \mathrm{keV}$ $[5,8,9]$. In the case of IGR J00291+5934 see Fig. 2 (right panel). These measurements permitted to perform an analysis of the pulsed fraction up to high X-ray energies and to confirm for the first time the increase of the pulsed fraction with energy in an accretion powered AMXP [5]. The latter could be succesfully interpreted in terms of Doppler effects on the exponentially cutoff Comptonized photons produced from the hot spot on the neutron star surface (see Fig. 3; left panel). Soft phase/time lags (low-energy pulses lag behind the high-energy pulses) with a complex energy dependence could also be measured and linked to the same effect. According to previous 

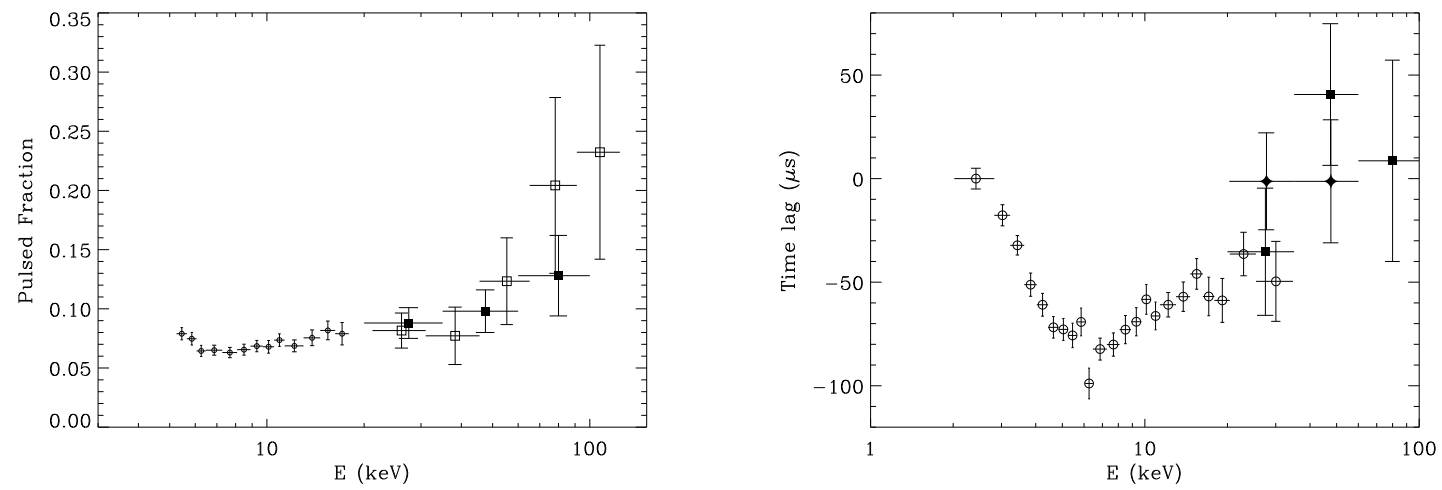

Figure 3: Left panel: The pulsed fraction of IGR J00291+5934 using pulsed/total flux measurements from PCA/HEXTE and JEM-X/ISGRI (open circles, PCA pulsed flux relative to JEM-X total flux; open squares, HEXTE pulsed flux relative to ISGRI total flux; filled squares, ISGRI pulsed flux relative to ISGRI total flux). The pulsed fraction gradually increases from $\sim 6 \%$ at $\sim 6 \mathrm{keV}$ to $\sim 12-20 \%$ at $\sim 100 \mathrm{keV}$ [5]. Right panel: IGR J00291+5934 time lags as a function of energy in the $2-100 \mathrm{keV}$ energy range combining RXTE/PCA (2-35 keV, open circles), RXTE/HEXTE (20-60 keV, filled diamonds), and INTEGRAL/ISGRI (20 - $100 \mathrm{keV}$, filled squares) measurements. The time lags first increase and then decrease slightly, saturating above $15 \mathrm{keV}$, and possibly reaching zero around $50 \mathrm{keV}$ [5].

measurements performed on the X-ray emission from SAX J1808.4-3658 and XTE J1751-350, the time lags revealed by INTEGRAL monotonically increased with energy and saturated at about 10-20 keV. The lags are most probably due to the different emission patterns of the blackbody and Comptonization components $[20,11,13]$ combined with the action of the Doppler effect. This interpretation is supported by the energy dependence of the lags, which grows until the contribution of the blackbody becomes negligible. At higher energies, the soft lags can evolve further. This is due to the fact that higher-energy photons suffer more scatterings and the latter produce notable variations in the emission pattern as a function of the energy (see e.g, $[8,9]$ and reference therein). An example of measured time lags in the AMXP IGR J00291+5934 is shown in Figure 3 (right panel). In this case, the time lags first increase and then decrease slightly, saturating above $15 \mathrm{keV}$, and possibly reaching zero around $50 \mathrm{keV}$ (see also [4]).

By using RXTE/PCA data for IGR J00291+5934 in outburst, it was possible to measure for the first time a decrease of the NS spin period while the source was accreting material [6]. This provided an evidence in favor of the so-called "recycling" scenario, according to which old neutron stars in LMXB become millisecond X-ray pulsars when they are spun-up to such periods by the transfer of angular momentum from the accreting matter. This result was broadly communicated to scientific community through an ESA press release and global coverage in the popular press (see http://www.esa.int/esaSC/SEMWSAA5QCE_index_0.html).

The complementary INTEGRAL and RXTE observations provide an opportunity to crosscalibrate the absolute timing of the two instruments. The cross-correlation of the folded solar system barycentered event lists collected during the outburst of IGR J17511-3057 from ISGRI (15$90 \mathrm{keV}, 60$ bins), RXTE/PCA (15-60 keV, 60 bins) and RXTE/HEXTE (15-90 keV, 60 bins) in the same energy band gave time shifts of $-49+/-114 \mu \mathrm{s}$, and $-69+/-49 \mu \mathrm{s}$, respectively $[15,5,8,9]$. INTEGRAL event times seemed to be $50 \mu \mathrm{s}$ ahead with respect to the RXTE ones. Note, that this is almost the value of $47 \mu$ s that has been added in the process of updating/regenerating the 
INTEGRAL time correlation files and used also to update the ISGRI User Manual. So, from an experimental point of view there is no justification to apply this shift of $47 \mu \mathrm{s}$ in the INTEGRAL correlation files, but within errors everything still matches. We can state that INTEGRAL ISGRI and RXTE PCA/HEXTE timing measurements are consistent in absolute sense at the $50 \mu$ s level. We have thus the absolute timing information to perform a confident timing analysis. However, only with the RXTE monitoring observations over long time spans (to establish orbital parameters) an ephemeris could be constructed and used for the INTEGRAL/ISGRI events data. A blind searches with INTEGRAL will not lead to establish the AMXP nature. One long XMM-Newton observation may establish the AMXP nature, but can not yield an ephemeris without a long monitoring program. Nowadays without monitoring observations and without sensitive X-ray instruments with ms-timing capabilities the discovery of new AMXPs is unlikely.

\section{The AMXP observed X-ray bursts}
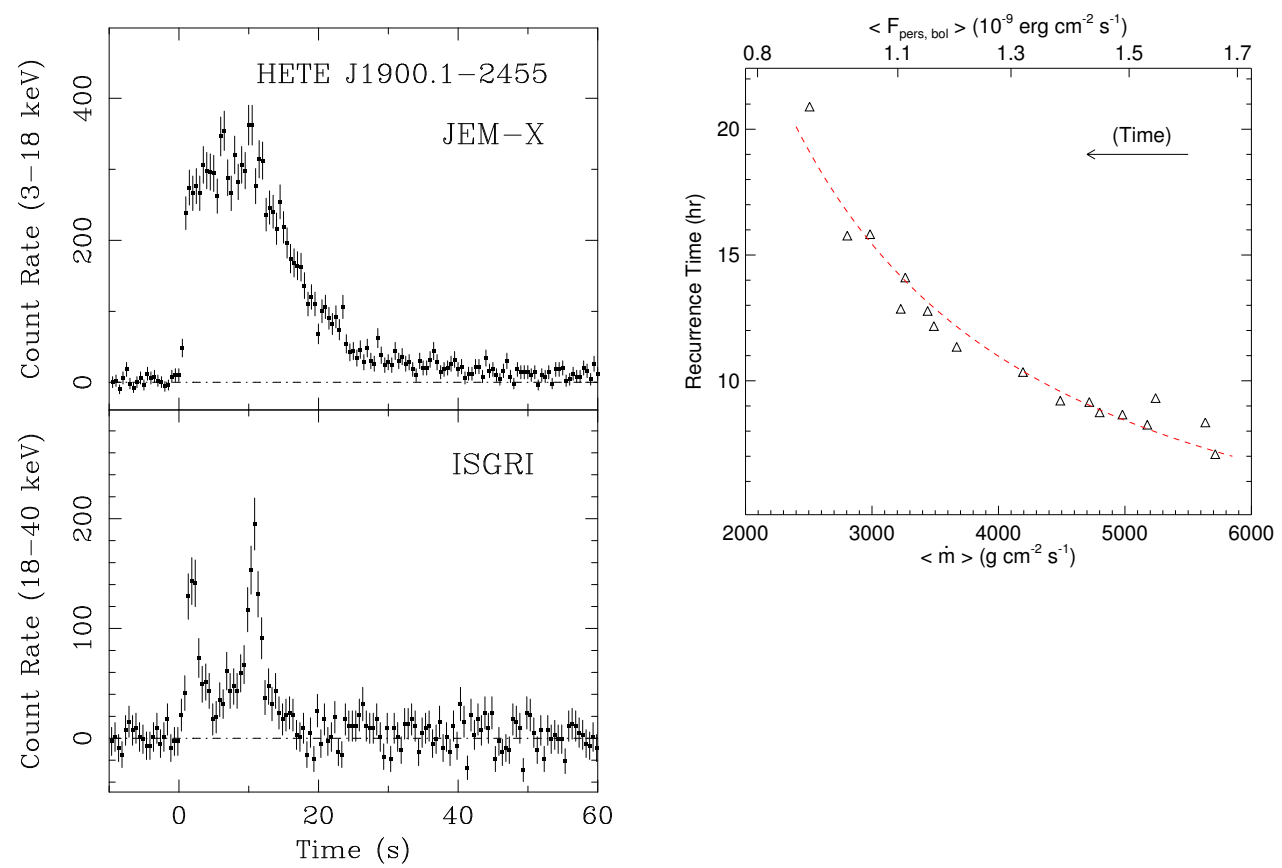

Figure 4: Left panel: The JEM-X (3-20 keV, upper panel) and IBIS/ISGRI (18-40 keV; lower panel) background subtracted lightcurve of a bright X-ray burst detected from HETE J1900.1-2455. The time bin is 0.5 s. At high-energy the burst shows strong evidence of photospheric radius expansion [7]. Right panel: Triangles in the figure represent the observed burst recurrence times shown as a function of local mass accretion rate (the corresponding flux is reported in the upper X-axis) for IGR J17511-3057. We also show the best-fit power-law model. The recurrence time increased with time roughly as $\left\langle F_{\text {pers,bol }}\right\rangle^{-1.1}[8]$.

INTEGRAL observed several type-I X-ray bursts from the sources HETE 1900.1-2455, Swift J1749.4-2807, IGR J17511-3057, and IGR J17498-2921 [7, 10, 8, 9]. Thermonuclear (type-I) X-ray bursts are produced by unstable burning of accreted matter on the NS surface. The spectrum from a few keV to higher energies can usually be well described by using a blackbody model with temperature $k T_{\mathrm{bb}} \approx 1-3 \mathrm{keV}$. The energy-dependent decay time of these bursts is attributed to the cooling of the NS photosphere and results in a gradual softening of the burst spectrum with time 
(see e.g., [16] for a review). Long ( 220 ks) uninterrupted INTEGRAL observations of AMXPs in outbursts permitted to detect series of type-I bursts and constrain accurately their recurrence time. In addition, the dependency of the recurrent time on accretion rate and the ignition depth could be measured by making use of combined observations carried out by INTEGRAL, RXTE, Swift, Chandra, and XMM-Newton. In all the above mentioned sources, the short burst profiles provided indication of hydrogen-poor material at ignition, suggesting either that the accreted material is hydrogen-deficient, or that the CNO metallicity is slightly higher than the solar value in the AMXPs. For instance, for IGR J17511-3057 (see Fig. 4 right panel) the variation of the burst recurrence time as a function of $\dot{m}$ (inferred from the X-ray flux) is much smaller than predicted by helium-ignition models [8].

\section{Summary}

INTEGRAL was able to observe at least one AMXP per year and discovered three objects belonging to this class. By combining high-energy INTEGRAL data with those obtained from RXTE, $X M M$-Newton, and Swift, it was possible to study the timing and bursts properties of the AMXPs in a wide energy range (0.3-150 keV). Our research group has been leading INTEGRAL observational campaign of AMXPs since the early operational phases. Our AMXP Target of Opportunity (ToO) proposals were approved during AO2-A10 cycles, and during the past few years our ToOs were triggered five times. We observed IGR J00291+5934 in 2004, HETE 1900.1-2455 in 2005, IGR J17511-3057 in 2009, IGR J17498-2921 in 2011, and Swift J1749.4-2807 in 2010. These observations were possible thanks to the prompt reactions to our requests from C. Winkler and the INTEGRAL staff, to whom we are grateful. We are also grateful to all TAC members who positively evaluated our research programs over years and permitted crucial advancements in the understanding of the physics of AMXPs.

\section{References}

[1] Chenevez, J., Kuulkers, E., Beckmann, V., et al. 2009, The Astronomers Telegram, 2235, 1

[2] Falanga, M., Soldi, S., Shaw, S., et al. 2007, The Astronomers Telegram, 1046, 1

[3] Falanga, M. 2008, in Cool Discs, Hot Flows: The Varying Faces of Accreting Compact Objects. ed. M. Axelsson (Melville, NY: AIP), AIP Conf. Ser. 1054, 157

[4] Falanga, M., \& Titarchuk, L. 2007, ApJ, 661, 1084

[5] Falanga, M., Bonnet-Bidaud, J. M., Poutanen, J., et al. 2005b, A\&A, 436, 647

[6] Falanga, M., Kuiper, L., Poutanen, J., et al. 2005a, A\&A, 444, 15
[7] Falanga, M., Poutanen, J., Bonning, E. W., et al. 2007, A\&A, 464, 1069

[8] Falanga, M., Kuiper, L., Poutanen, J., et al. 2012, A\&A, 529, 68

[9] Falanga, M., Kuiper, L., Poutanen, J., et al. 2012, A\&A, 545, 26

[10] Ferrigno, C., Bozzo, E., Falanga, M., 2011, A\&A, 525, 48

[11] Gierliński, M., \& Poutanen, J. 2005, MNRAS, 359, 1261

[12] Grebenev, S. A., Molkov, S. V., \& Sunyaev, R. A. 2005, The Astronomers Telegram, 446, 1

[13] Ibragimov, A., \& Poutanen, J. 2009, MNRAS, 400, 492

[14] King, A. R., Ritter, H., 1998, MNRAS, 293, L42 
[15] Kuiper, L., Hermsen, W., Walter, R.; Foschini, L., 2003, A\&A, 411, L31

[16] Lewin, W. H. G. L., van Paradijs, J., \& Taam, R. 1993, Sp. Sci. Rev., 62, 223

[17] Markwardt, C. B., Swank, J. H. Strohmayer, T. E. et al., 2002, ApJ, 575, L21

[18] Patruno, A., Watts, A. L., 2012, in Belloni T., Mendez M., Zhang C. M., eds., Timing neutron stars: pulsations, oscillations and explosions. Springer, New York (arXiv:1206.2727)

[19] Poutanen, J. 2006, Adv. Space Res., 38, 2697
[20] Poutanen, J., \& Gierliński, M. 2003, MNRAS, 343, 1301

[21] Poutanen, J., \& Svensson, R. 1996, ApJ, 470, 249

[22] Powell R., Haswell C. \& Falanga M., 2007, MNRAS, 374, 466

[23] Riggio, A. Burderi, L. Di Salvo, T., et al., 2011, A\&A, 531, 140

[24] Shaw, S. E. et al. 2005, A\&A, 432, 13

[25] Wijnands, R. 2006, in Trends in Pulsar Research, ed. J. A. Lowry (NY: Nova Science Publishers), 53 